\title{
Microdata Expenditure Analysis of Disaggregate Meat Products
}

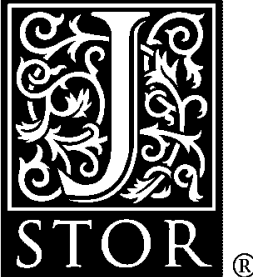

Rodolfo M. Nayga, Jr.

Review of Agricultural Economics, Vol. 17, No. 3. (Sep., 1995), pp. 275-285.

Stable URL:

http://links.jstor.org/sici?sici=1058-7195\%28199509\%2917\%3A3\%3C275\%3AMEAODM\%3E2.0.CO\%3B2-7

Review of Agricultural Economics is currently published by American Agricultural Economics Association.

Your use of the JSTOR archive indicates your acceptance of JSTOR's Terms and Conditions of Use, available at

http://www.jstor.org/about/terms.html. JSTOR's Terms and Conditions of Use provides, in part, that unless you have obtained prior permission, you may not download an entire issue of a journal or multiple copies of articles, and you may use content in the JSTOR archive only for your personal, non-commercial use.

Please contact the publisher regarding any further use of this work. Publisher contact information may be obtained at http://www.jstor.org/journals/aaea.html.

Each copy of any part of a JSTOR transmission must contain the same copyright notice that appears on the screen or printed page of such transmission.

The JSTOR Archive is a trusted digital repository providing for long-term preservation and access to leading academic journals and scholarly literature from around the world. The Archive is supported by libraries, scholarly societies, publishers, and foundations. It is an initiative of JSTOR, a not-for-profit organization with a mission to help the scholarly community take advantage of advances in technology. For more information regarding JSTOR, please contact support@jstor.org. 


\title{
MICRODATA EXPENDITURE ANALYSIS OF DISAGGREGATE MEAT PRODUCTS
}

\author{
Rodolfo M. Nayga, Jr.
}

Several studies have examined consumer attitudes and preferences toward meat products (e.g., Branson et al.; Skaggs et al.; Menkhaus et al.). Other analyses have identified sources of structural changes in U.S. meat consumption (e.g., Nyankori and Miller; Braschler; Chavas; Moschini and Meilke) and have examined effects of demographic factors on meat consumption (e.g., Buse and Salathe; Cox, Buse, and Alvarez; Blaylock and Smallwood; Nayga and Capps 1992). These studies, however, have focused on broad and aggregated commodities. Due to the degree of aggregation, considerable information about consumption patterns of the commodities is potentially lost. Consequently, some researchers (Eales and Unnevehr; Pudney; Nayga and Capps 1994) have suggested that a full understanding of meat consumption patterns requires analysis of a disaggregated products model. Simply put, little information exists on factors affecting disaggregate meat expenditures at the household level.

Recent studies that have focused on disaggregate meat products (e.g., Capps and Nayga 1990, 1991; Nayga and Capps 1994) have used time-series scanner data from supermarkets. A drawback of using scanner data is that it does not usually provide information about consumers' demographic characteristics. Recent interest in demographic effects has led researchers to the use of cross-sectional microdata. The use of household-level microdata is popular because it avoids the problem of aggregation over consumers and often provides a large and statistically-rich sample (Heien and Wessells).

Rodolfo M. Nayga, Jr. is an Assistant Professor, Department of Agricultural Economics and Marketing, Rutgers University.

The author wishes to thank anonymous journal reviewers and the editor for helpful comments and suggestions. Support for this research comes from the New Jersey Agricultural Experiment Station.
The use of household-level data, however, is not without problems. Normally, a researcher is confronted with a proportion of the sample with zero consumption or expenditure of the various commodities under consideration. Applied economists have relied on numerous estimation techniques (e.g., Tobit and Cragg models) to address this issue (Tobin; Cragg). This article deviates from previous crosssectional studies that have focused on meat expenditures or consumption by examining 14 meat products at a relatively disaggregate level using the 1992 Consumer Expenditure Survey of the Bureau of Labor Statistics. Moreover, the expenditures on these 14 meat products are modeled as an interrelated demand system based on the Quadratic Expenditure System and a twostep estimation procedure.

\section{Model Specification}

Data used for this study do not contain price information on the various disaggregate meat products. This is the main drawback of using survey data. Usually, analysts have addressed this issue by imputing a price variable from expenditure and quantity information. However, the data base used in this study does not contain quantity information. Another possible remedy to this problem is to use regional price indexes for each household in a particular region for each category of expenditure. However, Heien and Durham criticized this approach because it "invariably captures effects other than the intended ones" (p. 193). They also acknowledged that the price variable derived from this approach will be linearly dependent with the regional dummy variables since the price for any given product will be the same for all households in a given period. In addition, detailed price indexes are not available at the level of aggregation analyzed in 
this article. Consequently, no price variables are included in the empirical models.

An inherent assumption in this study is that relative prices remained constant over the 1992 survey period. This assumption is commonly used in studies that use microdata. Therefore, it is not possible to represent the expenditure patterns of the disaggregate meat products using a complete demand system. However, following Heien and Durham, an interrelated demand system based on the Quadratic Expenditure System (QES) is employed to examine the effect of various factors on 14 disaggregate meat expenditures.'

The QES in expenditure form with prices normalized at unity may be written as:

$$
\begin{aligned}
\mathrm{e}_{\mathrm{ih}} & =\gamma_{\mathrm{ih}}+\alpha_{\mathrm{i}}\left(\mathrm{Y}_{\mathrm{h}}-\sum_{\mathrm{j}} \gamma_{\mathrm{jh}}\right) \\
& +\beta_{\mathrm{i}}\left(\mathrm{Y}_{\mathrm{h}}-\sum_{\mathrm{j}} \gamma_{\mathrm{jh}}\right)^{2} \\
\mathrm{j} & =1, \ldots, \mathrm{n},
\end{aligned}
$$

where $e_{i h}$ is the expenditure on the $i^{\text {th }}$ good by the $h^{\text {th }}$ household, and $Y_{h}$ is total expenditure or income. $\beta_{i}$ represents a combination of several QES parameters.

To incorporate demographic variables, the QES model can be modified through the process of demographic translation (Pollak and Wales). Based on the model shown in equation (1), this technique gives the following specification:

$$
\begin{aligned}
\gamma_{\text {ih }} & =\delta_{i 0}+\sum_{k} \delta_{i k} D_{k h} \\
\mathrm{i} & =1, \ldots, \mathrm{n} \\
\mathrm{j} & =1, \ldots, \mathrm{s},
\end{aligned}
$$

where $\delta_{i 0}$ and $\delta_{i k}$ 's are parameters and $D_{k h}$ are demographic variables (s number of variables) for the $h^{\text {th }}$ household. Following Heien and Durham, equation (2) is substituted into equation (1) and then simplified to produce a demographically-augmented Quadratic Engel Curve (QEC) as follows:

$$
\begin{aligned}
\mathrm{e}_{\mathrm{ih}} & =\gamma_{\mathrm{ih}}+\alpha_{\mathrm{i}} \mathrm{Y}_{\mathrm{h}}+\beta_{\mathrm{i}} \mathrm{Y}_{\mathrm{h}}^{2} \\
\mathrm{i} & =1, \ldots, \mathrm{n} .
\end{aligned}
$$

\footnotetext{
'An interrelated demand system, as defined by Heien and Durham, is one that is estimated by a technique that takes cross-equation error covariances into account.
}

The system given in equation (3) is considered an approximation to a demand system (see Heien and Durham for more details).

The data contain numerous zero observations on expenditures for the 14 meat products. These zero observations reflect nonpurchases that may be due to sufficient household inventory, responses to market price, or to nonpreference (Cheng and Capps). If only observed positive purchases are used in estimating equation (3), ordinary least squares estimates of equation (3) will be inconsistent from selectivity bias. In addition, Haines, Guilkey, and Popkin emphasized that ignoring the two-step nature of food consumption decisions "may hamper understanding of true behavioral patterns, lead to erroneous conclusions, and generate incorrect policy recommendations" (p. 543). Consequently, this study employs a two-step procedure, similar to the technique used by Heien and Wessells, to estimate the censored multiple-equation model. The two-step estimator resulting from this procedure is asymptotically more efficient than other two-step estimators (Lee). This procedure involves the special case of a model in which the dependent variables are censored by a subset of unobservable latent variables (Heien and Wessells).

The first step in this procedure involves the estimation of probit regression models for each of the meat products under consideration. The dependent variables in these probit regression models are measured by a binary variable reflecting the decision to buy or not to buy the product. These models are then used to compute the inverse Mill's ratio $(\Gamma)$ for each household. These $\Gamma$ 's, as specified by Heckman, are derived for each equation and are then used as an instrument in the second-step estimation of the expenditure relations. follows:

The probit regressions are modeled as

$$
\begin{aligned}
\mathrm{P}_{\mathrm{ih}} & =f\left(\mathrm{D}_{1 \mathrm{~h}}, \ldots, \mathrm{D}_{\mathrm{sh}}, \mathrm{Y}_{\mathrm{h}}, \mathrm{Y}_{\mathrm{h}}^{2}\right) \\
\mathrm{i} & =1, \ldots, \mathrm{n},
\end{aligned}
$$

where $P_{\text {ih }}$ is 1 if the $h^{\text {th }}$ household consumes the $i^{\text {th }}$ item and 0 if the household does not consume the item in question. From the maximum 
likelihood estimates in equation (4), the inverse Mill's ratio for the household that consumes the item is derived as:

$$
\Gamma_{i h}=\frac{\pi\left(D_{1 h}, \ldots, D_{s h}, Y_{h}, Y_{h}^{2}\right)}{\pi\left(D_{1 h}, \ldots, D_{s h}, Y_{h}, Y_{h}^{2}\right)},
$$

where $\pi$ and $\Pi$ are the standard normal density and cumulative probability functions, respectively. The inverse Mill's ratio for those households who do not consume the item is computed as:

$$
\Gamma_{i h}=\frac{\pi\left(D_{1 h}, \ldots, D_{s h}, Y_{h}, Y_{h}^{2}\right)}{\left(1-\pi\left(D_{1 h}, \ldots, D_{s h}, Y_{h}, Y_{h}^{2}\right)\right)} .
$$

The inverse Mill's ratio for each equation is then used as an instrument in the estimation of equation (3) by specifying:

$$
\begin{aligned}
\mathrm{Y}_{\mathrm{ih}} & =\delta_{\mathrm{i} 0}+\sum_{\mathrm{k}} \delta_{\mathrm{ik}} \mathrm{D}_{\mathrm{kh}}+\theta_{\mathrm{i}} \Gamma_{\mathrm{ih}} \\
\mathrm{i} & =1, \ldots, \mathrm{n} .
\end{aligned}
$$

The specification for the expenditure equations is:

$$
\begin{aligned}
\mathrm{e}_{i h} & =\delta_{i}+\sum_{k} \delta_{i k} D_{k h}+\theta_{i} \Gamma_{i h} \\
& +\alpha_{i} Y_{i h}+\beta_{i} Y_{i h}^{2} \\
i & =1, \ldots, n .
\end{aligned}
$$

All $n$ commodity equations in equation (8) are estimated as a system using all observations in the sample. Each equation has the same set of regressors except for the inverse Mill's ratios. Consequently, the $\mathrm{n}$ equations are estimated using the Seemingly Unrelated Regression (SUR) technique to gain efficiency and to account for possible contemporaneous correlation among the disturbance terms. The description of the independent variables $\left(D_{k h}\right.$ 's) used in this study are presented in Table 1 . The dependent variables $\left(\mathrm{e}_{\mathrm{ih}}\right.$ 's) are the weekly household expenditures on 14 disaggregate meat products (see Table 2).

\section{Data}

The data are from the Diary component of the 1992 Consumer Expenditure Survey (CES) of the Bureau of Labor Statistics (United States
Department of Labor). The CES was designed to collect information on expenditures incurred by respondents during a survey week. The respondents are part of a national probability sample of households designed to represent the total civilian population. The eligible population includes all civilian noninstitutional persons (e.g., those living in houses, condominiums, or apartments) and all people residing in group quarters such as housing facilities for students and workers. Military personnel living on bases are not included (United States Department of Labor). The total number of households (diaries) in the survey was 11,412 . However, households that reported incomplete socioeconomic and demographic information were dropped from the analysis. In addition, households with negative incomes were deleted from the sample. Consequently, the number of households analyzed in the study was 10,359 . The mean weekly expenditures on the 14 disaggregate meat products for the reporting households are presented in Table 2. The means and standard deviations of the independent variables used in the analysis also are presented in Table 2 .

Deleting data for households that fail to report pertinent socioeconomic information may give rise to a potential self-selection problem. One way to assess the degree to which this problem may exist is by comparing the means of the variables used in the analyses with and without the deleted data (those observations with incomplete sociodemographic and economic information). The means of the variables with the deleted data are not significantly different from the means of the variables without the deleted data.

\section{Econometric Results}

A problem in multiple regression is multicollinearity. Based on the collinearity diagnostic tests conducted (Belsley, Kuh, and Welsch), no degrading collinearity problems are detected in the analyses. The SUR estimates of the QEC system are presented in Table $3 .^{2}$ Households without children spend $\$ 0.32$ less

${ }^{2}$ The results from the first step of the estimation procedure are not presented, but are available from the author upon request. 
Table 1. Independent Variables in the Model

\begin{tabular}{|c|c|c|}
\hline Variate & $\begin{array}{c}\text { Variable } \\
\text { Name }\end{array}$ & Description \\
\hline Children $^{\mathrm{a}}$ & Nochild & $\begin{array}{l}\text { Absence of children } \\
\text { Presence of children }\end{array}$ \\
\hline Earners & No_earnr & Number of earners in the household \\
\hline Region $^{\text {a.c }}$ & $\begin{array}{l}\text { Northeast } \\
\text { Midwest } \\
\text { South } \\
\text { West }\end{array}$ & $\begin{array}{l}\text { Household located in the Northeast } \\
\text { Household located in the Midwest } \\
\text { Household located in the South } \\
\text { Household located in the West }\end{array}$ \\
\hline Household Size & Fam_size & Number of persons in the household \\
\hline Season $^{a}$ & $\begin{array}{l}\text { Quarter1 } \\
\text { Quarter2 } \\
\text { Quarter3 } \\
\text { Quarter4 }\end{array}$ & $\begin{array}{l}\text { Household interviewed during the first quarter of the year } \\
\text { Household interviewed during the second quarter of the year } \\
\text { Household interviewed during the third quarter of the year } \\
\text { Household interviewed during the fourth quarter of the year }{ }^{b}\end{array}$ \\
\hline Age & $\begin{array}{l}\text { Age } \\
\text { Agesq }\end{array}$ & $\begin{array}{l}\text { Age in years of reference person } \\
\text { Square of age }\end{array}$ \\
\hline $\operatorname{Race}^{\mathrm{a}}$ & $\begin{array}{l}\text { White } \\
\text { Black } \\
\text { Othrace }\end{array}$ & $\begin{array}{l}\text { Reference person is white } \\
\text { Reference person is black } \\
\text { Reference person is of other race }\end{array}$ \\
\hline Education $^{a}$ & $\begin{array}{l}\text { Educ1 } \\
\text { Educ2 } \\
\text { Educ3 }\end{array}$ & $\begin{array}{l}\text { Reference person is less than a high school graduate } \\
\text { Reference person is less than a college graduate, but is a high } \\
\text { school graduate } \\
\text { Reference person is at least a college graduate }\end{array}$ \\
\hline Income & $\begin{array}{l}\text { Income } \\
\text { Incomesq }\end{array}$ & $\begin{array}{l}\text { Household income after taxes in the past } 12 \text { months }(\$) \\
\text { Square of income }\end{array}$ \\
\hline
\end{tabular}

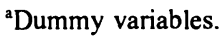

${ }^{b}$ Omitted category.

'The regions are comprised of the following states: Northeast - CT, MA, ME, NH, NJ, NY, PA, RI, and VT; Midwest - IA, IL, IN, KS, MI, MN, MO, NE, ND, OH, SD, and WI; South - AL, AR, DC, DE, FL, GA, KY, LA, MD, MS, NC, OK, SC, TN, TX, VA, and WV; West - AK, AZ, CA, CO, HI, ID, MT, NV, NM, OR, UT, WA, and WY.

on ground beef, $\$ 0.25$ less on steak, $\$ 0.14$ less on pork chops, and $\$ 0.15$ less on ham per week, but spend $\$ 0.68$ more on other pork, $\$ 0.15$ more on lunch meat, $\$ 0.64$ more on whole chicken, $\$ 1.10$ more on chicken parts, $\$ 0.20$ more on other poultry, $\$ 0.28$ more on shellfish, and $\$ 0.30$ more on finfish per week than households with children.

The number of earners in a household is positively related to expenditures on ground beef, beef roast, steak, lunch meat, and chicken parts, but is negatively related to the expenditures on whole chicken. In particular, the change in weekly household expenditures per unit change in the number of earners range from $(\$ 0.04)$ for whole chicken to $\$ 0.23$ for steak, ceteris paribus.

As a group, the regional variables are statistically significant in the steak, bacon, pork chops, lunch meat, other pork, whole chicken, chicken parts, and shellfish equations as indicated by the results of the likelihood ratio tests exhibited in Table 4. In particular, the results indicate that households located in the Northeast spend $\$ 0.15$ more on pork chops, $\$ 0.25$ more on other pork, $\$ 0.24$ more on lunch meat, and $\$ 0.15$ more on whole chicken per week, but spend $\$ 0.61$ less on chicken parts and $\$ 0.33$ less on shellfish per week than do households in the South (Table 3). On the other hand, households located in the Midwest spend $\$ 0.16$ more on ground beef, $\$ 0.06$ more on bacon, $\$ 0.19$ more on lunch meat, $\$ 0.54$ more on whole chicken, $\$ 0.44$ more on chicken parts and $\$ 0.57$ more on shellfish per week, but spend $\$ 0.22$ less on steak per week than do households in the South. Households in the West spend 
Table 2. Sample Statistics, 1992

\begin{tabular}{|c|c|c|}
\hline $\begin{array}{l}\text { Dependent } \\
\text { Variables }\end{array}$ & $\mathrm{N}^{\mathrm{a}}$ & $\begin{array}{c}\text { Mean }^{\mathrm{b}} \\
\text { (\$/Week) }\end{array}$ \\
\hline Ground Beef & 3,785 & 5.129 \\
\hline Roast & 1,102 & 8.002 \\
\hline Steak & 2,479 & 7.921 \\
\hline Bacon & 1,794 & 2.506 \\
\hline Pork Chops & 1,343 & 5.901 \\
\hline Ham & 1,868 & 5.460 \\
\hline Other Pork & 2,354 & 5.666 \\
\hline Lunch Meat & 4,517 & 4.246 \\
\hline Lamb-Mutton & 402 & 5.281 \\
\hline Whole Chicken & 1,158 & 4.002 \\
\hline Chicken Parts & 3,113 & 5.262 \\
\hline Other Poultry & 1,375 & 5.477 \\
\hline Shellfish & 640 & 9.080 \\
\hline Finfish & 1,347 & 6.648 \\
\hline $\begin{array}{c}\text { Independent } \\
\text { Variables }\end{array}$ & Mean $^{c}$ & $\begin{array}{c}\text { Standard } \\
\text { Deviation }^{\mathrm{d}}\end{array}$ \\
\hline Nochild & 0.55 & 0.49 \\
\hline No_earnr & 1.42 & 0.97 \\
\hline Northeast & 0.22 & 0.41 \\
\hline Midwest & 0.25 & 0.43 \\
\hline West & 0.23 & 0.42 \\
\hline Fam_size & 2.56 & 1.49 \\
\hline Quarterl & 0.24 & 0.42 \\
\hline Quarter2 & 0.22 & 0.41 \\
\hline Quarter3 & 0.21 & 0.41 \\
\hline Age & 46.56 & 0.40 \\
\hline Black & 0.11 & 0.30 \\
\hline Othrace & 0.04 & 0.19 \\
\hline Educl 1 & 0.19 & 0.39 \\
\hline Educ3 & 0.26 & 0.43 \\
\hline Income & $26,362.01$ & $26,013.32$ \\
\hline
\end{tabular}

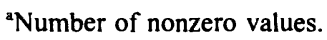

'Mean weekly household expenditure based on nonzero observations.

'Mean based on the whole sample $(\mathrm{N}=10,359)$.

${ }^{\mathrm{d}}$ Standard deviation based on the whole sample $(\mathrm{N}=10,359)$.

$\$ 0.39$ more on whole chicken and $\$ 0.12$ more on other poultry per week, but spend $\$ 0.31$ less on shellfish per week than do households in the South.

Household size is also a significant factor in all of the equations. Specifically, household size is positively related to expenditures on ground beef, beef roast, steak, bacon, pork chops, ham, other pork, lunch meat, lambmutton, and finfish, but is negatively related to expenditures on whole chicken, chicken parts, other poultry, and shellfish. The change in weekly household expenditures per unit change in household size range from (\$0.05) for other poultry to $\$ 0.39$ for ground beef, ceteris paribus. Household size elasticities are presented in Table 5. These estimates are in the inelastic range, with the exception of those for lamb-mutton, whole chicken, and shellfish.

Seasonality is a significant factor in the ground beef, ham, whole chicken, chicken parts, other poultry, and shellfish equations (Table 4). In particular, weekly ground beef expenditures are $\$ 0.24$ and $\$ 0.15$ higher during the second and third quarters of the year, respectively, compared to the fourth quarter of the year (Table 3). Ham, whole chicken, and chicken parts expenditures, however, are lower during the first three quarters of the year than during the last quarter of the year. This result is expected because of the holiday season. Compared to the fourth quarter of the year, weekly expenditures are $\$ 0.21$ lower for other poultry during the first quarter and \$0.17 and \$0.14 lower for finfish during the first and third quarters, respectively, but $\$ 0.31$ higher for shellfish during the third quarter of the year.

Age is also significantly related to expenditures on various meat products. Expenditures on ground beef, beef roast, steak, bacon, pork chops, and ham initially increase with age, and then decline as suggested by the significant positive and negative signs of the age and agesq coefficients. However, expenditures on other pork, whole chicken, chicken parts, other poultry, and finfish initially decrease with age followed by later increases. These results may reflect the change in meat expenditure patterns of households due to changing health concerns that tend to increase with age. These results may also be consistent with age-related changes in nutritional requirements, physiology, and demand for convenience.

As a group, the race variables are statistically significant in the ground beef, bacon, pork chops, whole chicken, chicken parts, shellfish, and finfish equations based on the likelihood ratio tests in Table 4. In particular, households headed by blacks spend $\$ 0.27$ less on steak, $\$ 0.28$ less on whole chicken, $\$ 0.77$ less on chicken parts, and $\$ 0.23$ less on shellfish per 
Table 3. SUR Estimates of the 14-Good QEC System

\begin{tabular}{|c|c|c|c|c|c|}
\hline Variable & $\begin{array}{c}\text { Ground } \\
\text { Beef }\end{array}$ & Roast & Steak & Bacon & $\begin{array}{l}\text { Pork } \\
\text { Chops }\end{array}$ \\
\hline Intercept & $\begin{array}{c}-0.548^{*} \\
(0.273)\end{array}$ & $\begin{array}{l}-1.146^{*} \\
(0.257)\end{array}$ & $\begin{array}{c}-0.841^{*} \\
(0.418)\end{array}$ & $\begin{array}{l}-0.314^{*} \\
(0.109)\end{array}$ & $\begin{array}{l}-0.122 \\
(0.197)\end{array}$ \\
\hline Nochild & $\begin{array}{l}-0.319^{*} \\
(0.091)\end{array}$ & $\begin{array}{c}0.040 \\
(0.085)\end{array}$ & $\begin{array}{l}-0.254^{*} \\
(0.139)\end{array}$ & $\begin{array}{c}0.025 \\
(0.037)\end{array}$ & $\begin{array}{l}-0.140^{*} \\
(0.066)\end{array}$ \\
\hline No_earnr & $\begin{array}{c}0.071^{*} \\
(0.040)\end{array}$ & $\begin{array}{c}0.142^{*} \\
(0.038)\end{array}$ & $\begin{array}{c}0.226^{*} \\
(0.061)\end{array}$ & $\begin{array}{l}-0.018 \\
(0.016)\end{array}$ & $\begin{array}{c}0.036 \\
(0.029)\end{array}$ \\
\hline Northeast & $\begin{array}{c}0.044 \\
(0.084)\end{array}$ & $\begin{array}{c}0.025 \\
(0.079)\end{array}$ & $\begin{array}{c}0.155 \\
(0.129)\end{array}$ & $\begin{array}{l}-0.052 \\
(0.033)\end{array}$ & $\begin{array}{c}0.153^{*} \\
(0.060)\end{array}$ \\
\hline Midwest & $\begin{array}{c}0.159^{*} \\
(0.080)\end{array}$ & $\begin{array}{l}-0.070 \\
(0.075)\end{array}$ & $\begin{array}{l}-0.223^{*} \\
(0.123)\end{array}$ & $\begin{array}{c}0.055^{*} \\
(0.032)\end{array}$ & $\begin{array}{c}0.018 \\
(0.058)\end{array}$ \\
\hline West & $\begin{array}{c}0.059 \\
(0.083)\end{array}$ & $\begin{array}{l}-0.042 \\
(0.078)\end{array}$ & $\begin{array}{c}0.040 \\
(0.127)\end{array}$ & $\begin{array}{l}-0.021 \\
(0.033)\end{array}$ & $\begin{array}{l}-0.026 \\
(0.060)\end{array}$ \\
\hline Fam_size & $\begin{array}{c}0.385^{*} \\
(0.031)\end{array}$ & $\begin{array}{c}0.207^{*} \\
(0.030)\end{array}$ & $\begin{array}{c}0.246^{*} \\
(0.048)\end{array}$ & $\begin{array}{c}0.119^{*} \\
(0.013)\end{array}$ & $\begin{array}{c}0.112^{*} \\
(0.023)\end{array}$ \\
\hline Quarterl ${ }^{\circ}$ & $\begin{array}{c}0.067 \\
(0.079)\end{array}$ & $\begin{array}{l}-0.008 \\
(0.075)\end{array}$ & $\begin{array}{l}-0.009 \\
(0.121)\end{array}$ & $\begin{array}{l}-0.050 \\
(0.031)\end{array}$ & $\begin{array}{c}0.065 \\
(0.057)\end{array}$ \\
\hline Quarter2 & $\begin{array}{c}0.243^{*} \\
(0.081)\end{array}$ & $\begin{array}{l}-0.056 \\
(0.076)\end{array}$ & $\begin{array}{c}0.036 \\
(0.125)\end{array}$ & $\begin{array}{l}-0.012 \\
(0.032)\end{array}$ & $\begin{array}{c}0.016 \\
(0.059)\end{array}$ \\
\hline Quarter3 & $\begin{array}{c}0.148^{*} \\
(0.082)\end{array}$ & $\begin{array}{l}-0.023 \\
(0.077)\end{array}$ & $\begin{array}{c}0.160 \\
(0.126)\end{array}$ & $\begin{array}{l}-0.0003 \\
(0.033)\end{array}$ & $\begin{array}{c}0.003 \\
(0.059)\end{array}$ \\
\hline Age & $\begin{array}{c}0.044^{*} \\
(0.010)\end{array}$ & $\begin{array}{c}0.038^{*} \\
(0.010)\end{array}$ & $\begin{array}{c}0.062^{*} \\
(0.016)\end{array}$ & $\begin{array}{c}0.015^{*} \\
(0.004)\end{array}$ & $\begin{array}{c}0.014^{*} \\
(0.007)\end{array}$ \\
\hline Agesq & $\begin{array}{l}-0.0004^{*} \\
(0.0001)\end{array}$ & $\begin{array}{l}-0.0002^{*} \\
(0.0001)\end{array}$ & $\begin{array}{l}-0.001^{\circ} \\
(0.0001)\end{array}$ & $\begin{array}{l}-0.0001^{*} \\
(0.00004)\end{array}$ & $\begin{array}{l}-0.0001^{*} \\
(0.00007)\end{array}$ \\
\hline Black & $\begin{array}{c}0.012 \\
(0.098)\end{array}$ & $\begin{array}{l}-0.009 \\
(0.093)\end{array}$ & $\begin{array}{l}-0.268^{*} \\
(0.151)\end{array}$ & $\begin{array}{c}0.136^{*} \\
(0.039)\end{array}$ & $\begin{array}{c}0.297^{*} \\
(0.071)\end{array}$ \\
\hline Othrace & $\begin{array}{l}-0.522^{*} \\
(0.153)\end{array}$ & $\begin{array}{l}-0.148 \\
(0.144)\end{array}$ & $\begin{array}{c}0.121 \\
(0.234)\end{array}$ & $\begin{array}{c}0.032 \\
(0.061)\end{array}$ & $\begin{array}{l}-0.009 \\
(0.110)\end{array}$ \\
\hline Educl & $\begin{array}{c}0.043 \\
(0.083)\end{array}$ & $\begin{array}{l}-0.005 \\
(0.078)\end{array}$ & $\begin{array}{c}0.061 \\
(0.127)\end{array}$ & $\begin{array}{c}0.003 \\
(0.033)\end{array}$ & $\begin{array}{c}0.153^{*} \\
(0.060)\end{array}$ \\
\hline Educ3 & $\begin{array}{l}-0.314^{*} \\
(0.073)\end{array}$ & $\begin{array}{r}0.0001 \\
(0.068)\end{array}$ & $\begin{array}{l}-0.279^{*} \\
(0.111)\end{array}$ & $\begin{array}{l}-0.110^{*} \\
(0.029)\end{array}$ & $\begin{array}{l}-0.119^{*} \\
(0.052)\end{array}$ \\
\hline Income & $\begin{array}{c}6.6 \mathrm{E}-6^{*} \\
(2.6 \mathrm{E}-6)\end{array}$ & $\begin{array}{c}2.4 \mathrm{E}-6 \\
(2.5 \mathrm{E}-6)\end{array}$ & $\begin{array}{c}9.1 \mathrm{E}-6^{*} \\
(4.0 \mathrm{E}-6)\end{array}$ & $\begin{array}{c}6.3 \mathrm{E}-7 \\
(1.0 \mathrm{E}-6)\end{array}$ & $\begin{array}{c}4.4 \mathrm{E}-6 * \\
(1.9 \mathrm{E}-6)\end{array}$ \\
\hline Incomesq & $\begin{array}{l}-6.3 \mathrm{E}-11^{*} \\
(2.4 \mathrm{E}-11)\end{array}$ & $\begin{array}{l}-2.8 \mathrm{E}-11 \\
(2.3 \mathrm{E}-11)\end{array}$ & $\begin{array}{l}-2.1 \mathrm{E}-11 \\
(3.7 \mathrm{E}-11)\end{array}$ & $\begin{array}{l}-4.9 \mathrm{E}-12 \\
(9.8 \mathrm{E}-12)\end{array}$ & $\begin{array}{l}-3.5 \mathrm{E}-11^{*} \\
(1.7 \mathrm{E}-11)\end{array}$ \\
\hline$\Gamma_{\text {ih }}$ & $\begin{array}{c}3.7 \mathrm{E}-8 \\
(2.7 \mathrm{E}-8)\end{array}$ & $\begin{array}{c}1.9 \mathrm{E}-15 \\
(3.2 \mathrm{E}-15)\end{array}$ & $\begin{array}{l}-2.5 E-7 \\
(4.4 E-7)\end{array}$ & $\begin{array}{l}-1.9 \mathrm{E}-13 \\
(3.2 \mathrm{E}-13)\end{array}$ & $\begin{array}{c}3.5 \mathrm{E}-14 \\
(4.1 \mathrm{E}-14)\end{array}$ \\
\hline
\end{tabular}

"Statistically significant at the 0.05 level.

Note: Standard errors are in parentheses. These estimates were derived using the SUR (joint generalized least squares) technique in the second stage of the estimation procedure. 
Table 3, Cont'd. SUR Estimates of the 14-Good QEC System

\begin{tabular}{|c|c|c|c|c|c|}
\hline Variable & Ham & $\begin{array}{l}\text { Other } \\
\text { Pork }\end{array}$ & $\begin{array}{c}\text { Lunch } \\
\text { Meat }\end{array}$ & $\begin{array}{l}\text { Lamb- } \\
\text { Mutton }^{2}\end{array}$ & $\begin{array}{l}\text { Whole } \\
\text { Chicken }\end{array}$ \\
\hline Intercept & $\begin{array}{l}-0.743^{*} \\
(0.273)\end{array}$ & $\begin{array}{c}4.478^{*} \\
(0.412)\end{array}$ & $\begin{array}{c}1.390^{*} \\
(0.302)\end{array}$ & $\begin{array}{c}0.087 \\
(0.137)\end{array}$ & $\begin{array}{c}4.130^{*} \\
(0.166)\end{array}$ \\
\hline Nochild & $\begin{array}{l}-0.154^{*} \\
(0.091)\end{array}$ & $\begin{array}{c}0.678^{*} \\
(0.111)\end{array}$ & $\begin{array}{c}0.150^{*} \\
(0.088)\end{array}$ & $\begin{array}{c}0.063 \\
(0.042)\end{array}$ & $\begin{array}{c}0.641^{*} \\
(0.046)\end{array}$ \\
\hline No_earnr & $\begin{array}{c}0.060 \\
(0.040)\end{array}$ & $\begin{array}{c}0.008 \\
(0.047)\end{array}$ & $\begin{array}{c}0.128^{*} \\
(0.038)\end{array}$ & $\begin{array}{l}-0.025 \\
(0.018)\end{array}$ & $\begin{array}{l}-0.043^{*} \\
(0.020)\end{array}$ \\
\hline Northeast & $\begin{array}{c}0.074 \\
(0.084)\end{array}$ & $\begin{array}{c}0.252^{*} \\
(0.101)\end{array}$ & $\begin{array}{c}0.241^{*} \\
(0.080)\end{array}$ & $\begin{array}{c}0.020 \\
(0.038)\end{array}$ & $\begin{array}{r}0.147^{*} \\
(0.041)\end{array}$ \\
\hline Midwest & $\begin{array}{l}-0.032 \\
(0.080)\end{array}$ & $\begin{array}{c}0.126 \\
(0.097)\end{array}$ & $\begin{array}{c}0.190^{\circ} \\
(0.077)\end{array}$ & $\begin{array}{l}-0.004 \\
(0.038)\end{array}$ & $\begin{array}{c}0.538^{*} \\
(0.041)\end{array}$ \\
\hline West & $\begin{array}{l}-0.066 \\
(0.083)\end{array}$ & $\begin{array}{l}-0.070 \\
(0.099)\end{array}$ & $\begin{array}{c}0.126 \\
(0.079)\end{array}$ & $\begin{array}{l}-0.033 \\
(0.038)\end{array}$ & $\begin{array}{c}0.391^{\circ} \\
(0.041)\end{array}$ \\
\hline Fam_size & $\begin{array}{c}0.123^{*} \\
(0.031)\end{array}$ & $\begin{array}{c}0.152^{*} \\
(0.039)\end{array}$ & $\begin{array}{c}0.254^{*} \\
(0.030)\end{array}$ & $\begin{array}{c}0.089^{*} \\
(0.015)\end{array}$ & $\begin{array}{l}-0.195^{*} \\
(0.017)\end{array}$ \\
\hline Quarterl & $\begin{array}{l}-0.276^{*} \\
(0.079)\end{array}$ & $\begin{array}{l}-0.018 \\
(0.094)\end{array}$ & $\begin{array}{c}0.045 \\
(0.076)\end{array}$ & $\begin{array}{c}0.029 \\
(0.036)\end{array}$ & $\begin{array}{l}-0.490^{*} \\
(0.040)\end{array}$ \\
\hline Quarter2 & $\begin{array}{c}-0.136^{*} \\
(0.081)\end{array}$ & $\begin{array}{c}0.149 \\
(0.097)\end{array}$ & $\begin{array}{c}0.008 \\
(0.078)\end{array}$ & $\begin{array}{l}-0.005 \\
(0.037)\end{array}$ & $\begin{array}{l}-0.232^{*} \\
(0.040)\end{array}$ \\
\hline Quarter3 & $\begin{array}{l}-0.228^{*} \\
(0.082)\end{array}$ & $\begin{array}{c}0.162 \\
(0.099)\end{array}$ & $\begin{array}{c}0.097 \\
(0.079)\end{array}$ & $\begin{array}{l}-0.050 \\
(0.038)\end{array}$ & $\begin{array}{l}-0.282^{*} \\
(0.041)\end{array}$ \\
\hline Age & $\begin{array}{c}0.055^{*} \\
(0.010)\end{array}$ & $\begin{array}{c}-0.103^{*} \\
(0.013)\end{array}$ & $\begin{array}{c}0.006 \\
(0.010)\end{array}$ & $\begin{array}{l}-0.005 \\
(0.005)\end{array}$ & $\begin{array}{l}-0.061^{*} \\
(0.005)\end{array}$ \\
\hline Agesq & $\begin{array}{l}-0.0004^{*} \\
(0.0001)\end{array}$ & $\begin{array}{c}0.0008^{*} \\
(0.0001)\end{array}$ & $\begin{array}{l}-0.0001 \\
(0.0001)\end{array}$ & $\begin{array}{c}0.00006 \\
(0.00005)\end{array}$ & $\begin{array}{c}0.0004^{*} \\
(0.00005)\end{array}$ \\
\hline Black & $\begin{array}{l}-0.098 \\
(0.098)\end{array}$ & $\begin{array}{c}0.080 \\
(0.120)\end{array}$ & $\begin{array}{l}-0.033 \\
(0.094)\end{array}$ & $\begin{array}{c}0.086^{*} \\
(0.045)\end{array}$ & $\begin{array}{l}-0.276^{*} \\
(0.050)\end{array}$ \\
\hline Othrace & $\begin{array}{l}-0.273^{*} \\
(0.153)\end{array}$ & $\begin{array}{c}0.329^{\circ} \\
(0.183)\end{array}$ & $\begin{array}{c}0.179 \\
(0.147)\end{array}$ & $\begin{array}{c}0.050 \\
(0.069)\end{array}$ & $\begin{array}{l}-0.191^{*} \\
(0.075)\end{array}$ \\
\hline Educl & $\begin{array}{l}-0.037 \\
(0.083)\end{array}$ & $\begin{array}{c}0.086 \\
(0.099)\end{array}$ & $\begin{array}{c}0.031 \\
(0.079)\end{array}$ & $\begin{array}{c}0.049 \\
(0.069)\end{array}$ & $\begin{array}{l}-0.307^{\circ} \\
(0.041)\end{array}$ \\
\hline Educ3 & $\begin{array}{l}-0.198^{*} \\
(0.073)\end{array}$ & $\begin{array}{c}0.428^{*} \\
(0.092)\end{array}$ & $\begin{array}{l}-0.006 \\
(0.071)\end{array}$ & $\begin{array}{c}0.048 \\
(0.033)\end{array}$ & $\begin{array}{c}0.050 \\
(0.035)\end{array}$ \\
\hline Income & $\begin{array}{c}2.6 \mathrm{E}-6 \\
(2.6 \mathrm{E}-6)\end{array}$ & $\begin{array}{l}-1.2 \mathrm{E}-5^{*} \\
(2.7 \mathrm{E}-6)\end{array}$ & $\begin{array}{c}2.7 \mathrm{E}-6 \\
(2.2 \mathrm{E}-6)\end{array}$ & $\begin{array}{l}-1.8 \mathrm{E}-7 \\
(1.0 \mathrm{E}-6)\end{array}$ & $\begin{array}{l}-2.5 \mathrm{E}-6^{*} \\
(1.1 \mathrm{E}-6)\end{array}$ \\
\hline Incomesq & $\begin{array}{l}-7.8 \mathrm{E}-12 \\
(2.4 \mathrm{E}-11)\end{array}$ & $\begin{array}{c}1.2 \mathrm{E}-10^{\circ} \\
(2.3 \mathrm{E}-11)\end{array}$ & $\begin{array}{l}-5.3 \mathrm{E}-12 \\
(1.8 \mathrm{E}-11)\end{array}$ & $\begin{array}{c}5.1 \mathrm{E}-12 \\
(8.8 \mathrm{E}-12)\end{array}$ & $\begin{array}{c}1.6 \mathrm{E}-11^{\circ} \\
(9.4 \mathrm{E}-12)\end{array}$ \\
\hline$\Gamma_{\text {ih }}$ & $\begin{array}{l}-1.7 \mathrm{E}-6 \\
(1.3 \mathrm{E}-5)\end{array}$ & $\begin{array}{l}-0.362^{\circ} \\
(0.015)\end{array}$ & $\begin{array}{l}-0.794^{*} \\
(0.042)\end{array}$ & $\begin{array}{l}-0.0003^{*} \\
(0.00007)\end{array}$ & $\begin{array}{l}-0.120^{*} \\
(0.002)\end{array}$ \\
\hline
\end{tabular}

Includes lamb, mutton, goat, and game.

'Statistically significant at the 0.05 level.

Note: Standard errors are in parentheses. These estimates were derived using the SUR technique in the second stage of the estimation procedure. 
282 REVIEW OF AGRICULTURAL ECONOMICS, Vol. 17, No. 3, September 1995

Table 3, Cont'd. SUR Estimates of the 14-Good QEC System

\begin{tabular}{|c|c|c|c|c|}
\hline Variable & $\begin{array}{c}\text { Chicken } \\
\text { Parts }\end{array}$ & $\begin{array}{l}\text { Other } \\
\text { Poultry }\end{array}$ & Shellfish & Finfish \\
\hline Intercept & $\begin{array}{c}7.950^{*} \\
(0.310)\end{array}$ & $\begin{array}{c}3.391^{*} \\
(0.289)\end{array}$ & $\begin{array}{c}3.977^{*} \\
(0.318)\end{array}$ & $\begin{array}{c}2.219^{*} \\
(0.332)\end{array}$ \\
\hline Nochild & $\begin{array}{c}1.101^{*} \\
(0.091)\end{array}$ & $\begin{array}{c}0.196^{*} \\
(0.074)\end{array}$ & $\begin{array}{c}0.279^{*} \\
(0.094)\end{array}$ & $\begin{array}{c}0.303^{*} \\
(0.091)\end{array}$ \\
\hline No_earnr & $\begin{array}{c}0.097^{*} \\
(0.038)\end{array}$ & $\begin{array}{c}0.018 \\
(0.032)\end{array}$ & $\begin{array}{l}-0.057 \\
(0.041)\end{array}$ & $\begin{array}{c}0.054 \\
(0.040)\end{array}$ \\
\hline Northeast & $\begin{array}{l}-0.606^{*} \\
(0.085)\end{array}$ & $\begin{array}{l}-0.011 \\
(0.067)\end{array}$ & $\begin{array}{l}-0.333^{*} \\
(0.088)\end{array}$ & $\begin{array}{c}0.063 \\
(0.085)\end{array}$ \\
\hline Midwest & $\begin{array}{c}0.442^{*} \\
(0.078)\end{array}$ & $\begin{array}{c}0.042 \\
(0.064)\end{array}$ & $\begin{array}{c}0.568^{*} \\
(0.087)\end{array}$ & $\begin{array}{c}0.015 \\
(0.079)\end{array}$ \\
\hline West & $\begin{array}{c}0.022 \\
(0.080)\end{array}$ & $\begin{array}{c}0.120^{*} \\
(0.067)\end{array}$ & $\begin{array}{l}-0.309^{*} \\
(0.086)\end{array}$ & $\begin{array}{l}-0.118 \\
(0.083)\end{array}$ \\
\hline Fam_size & $\begin{array}{l}-0.115^{*} \\
(0.031)\end{array}$ & $\begin{array}{l}-0.047^{*} \\
(0.026)\end{array}$ & $\begin{array}{l}-0.254^{*} \\
(0.035)\end{array}$ & $\begin{array}{c}0.061^{*} \\
(0.032)\end{array}$ \\
\hline Quarterl & $\begin{array}{l}-0.643^{*} \\
(0.078)\end{array}$ & $\begin{array}{l}-0.209^{*} \\
(0.063)\end{array}$ & $\begin{array}{l}-0.067 \\
(0.082)\end{array}$ & $\begin{array}{l}-0.171^{*} \\
(0.078)\end{array}$ \\
\hline Quarter2 & $\begin{array}{l}-0.389^{*} \\
(0.079)\end{array}$ & $\begin{array}{l}-0.055 \\
(0.067)\end{array}$ & $\begin{array}{l}-0.012 \\
(0.083)\end{array}$ & $\begin{array}{l}-0.046 \\
(0.080)\end{array}$ \\
\hline Quarter3 & $\begin{array}{l}-0.419^{*} \\
(0.080)\end{array}$ & $\begin{array}{l}-0.079 \\
(0.067)\end{array}$ & $\begin{array}{c}0.314^{*} \\
(0.087)\end{array}$ & $\begin{array}{l}-0.136^{*} \\
(0.081)\end{array}$ \\
\hline Age & $\begin{array}{l}-0.119^{*} \\
(0.010)\end{array}$ & $\begin{array}{l}-0.075^{*} \\
(0.010)\end{array}$ & $\begin{array}{l}-0.026^{*} \\
(0.010)\end{array}$ & $\begin{array}{l}-0.046^{*} \\
(0.011)\end{array}$ \\
\hline Agesq & $\begin{array}{c}0.0009^{*} \\
(0.0001)\end{array}$ & $\begin{array}{c}0.0006^{*} \\
(0.00009)\end{array}$ & $\begin{array}{c}0.0001 \\
(0.0001)\end{array}$ & $\begin{array}{c}0.0003^{*} \\
(0.0001)\end{array}$ \\
\hline Black & $\begin{array}{l}-0.767^{*} \\
(0.097)\end{array}$ & $\begin{array}{c}0.015 \\
(0.079)\end{array}$ & $\begin{array}{l}-0.229^{*} \\
(0.102)\end{array}$ & $\begin{array}{c}0.083 \\
(0.099)\end{array}$ \\
\hline Othrace & $\begin{array}{l}-0.236 \\
(0.148)\end{array}$ & $\begin{array}{l}-0.037 \\
(0.122)\end{array}$ & $\begin{array}{c}0.437^{*} \\
(0.162)\end{array}$ & $\begin{array}{c}1.469^{*} \\
(0.151)\end{array}$ \\
\hline Educl & $\begin{array}{l}-0.253^{*} \\
(0.080)\end{array}$ & $\begin{array}{c}0.150^{*} \\
(0.067)\end{array}$ & $\begin{array}{c}1.313^{*} \\
(0.080)\end{array}$ & $\begin{array}{c}0.101 \\
(0.082)\end{array}$ \\
\hline Educ3 & $\begin{array}{c}0.052 \\
(0.070)\end{array}$ & $\begin{array}{c}0.034 \\
(0.058)\end{array}$ & $\begin{array}{l}-0.583 \\
(0.080)\end{array}$ & $\begin{array}{c}0.024 \\
(0.072)\end{array}$ \\
\hline Income & $\begin{array}{l}-7.1 \mathrm{E}-6^{*} \\
(2.3 \mathrm{E}-6)\end{array}$ & $\begin{array}{l}-5.1 \mathrm{E}-6^{*} \\
(1.9 \mathrm{E}-6)\end{array}$ & $\begin{array}{l}-2.5 \mathrm{E}-5^{*} \\
(2.5 \mathrm{E}-6)\end{array}$ & $\begin{array}{l}-3.8 \mathrm{E}-6^{*} \\
(2.2 \mathrm{E}-6)\end{array}$ \\
\hline Incomesq & $\begin{array}{c}5.7 \mathrm{E}-11^{*} \\
(1.9 \mathrm{E}-11)\end{array}$ & $\begin{array}{c}6.1 \mathrm{E}-11^{*} \\
(1.5 \mathrm{E}-11)\end{array}$ & $\begin{array}{c}2.7 \mathrm{E}-10^{*} \\
(2.0 \mathrm{E}-11)\end{array}$ & $\begin{array}{c}8.3 \mathrm{E}-11^{*} \\
(1.8 \mathrm{E}-11)\end{array}$ \\
\hline$\Gamma_{\text {in }}$ & $\begin{array}{l}-1.679^{*} \\
(0.032)\end{array}$ & $\begin{array}{l}-0.067^{*} \\
(0.003)\end{array}$ & $\begin{array}{l}-0.044^{*} \\
(0.002)\end{array}$ & $\begin{array}{l}-0.045^{*} \\
(0.002)\end{array}$ \\
\hline
\end{tabular}

"Statistically significant at the 0.05 level.

Note: Standard errors are in parentheses. These estimates were derived using the SUR technique in the second stage of the estimation procedure. 
Table 4. Likelihood Ratio Test Statistics for Region, Season, Race, and Education Variables (F Values)

\begin{tabular}{lcccc}
\hline Equation & Region & Season & Race & Education \\
\hline Ground Beef & 1.350 & $3.242^{*}$ & $5.837^{*}$ & $10.383^{*}$ \\
Roast & 0.559 & 0.197 & 0.526 & 0.002 \\
Steak & $2.895^{*}$ & 0.687 & 1.759 & $3.681^{*}$ \\
Bacon & $3.404^{*}$ & 0.991 & $5.926^{*}$ & $7.535^{*}$ \\
Pork Chops & $3.132^{*}$ & 0.503 & $8.788^{*}$ & $7.491^{*}$ \\
Ham & 0.898 & $4.698^{*}$ & 1.988 & $3.695^{*}$ \\
Other Pork & $3.617^{*}$ & 1.751 & 1.747 & $10.631^{*}$ \\
Lunch Meat & $3.542^{*}$ & 0.589 & 0.839 & 0.093 \\
Lamb-Mutton & 0.600 & 1.236 & 1.969 & 1.584 \\
Whole Chicken & $64.414^{*}$ & $49.609^{*}$ & $18.138^{*}$ & $32.593^{*}$ \\
Chicken Parts & $43.628^{*}$ & $24.033^{*}$ & $31.729^{*}$ & $6.086^{*}$ \\
Other Poultry & 1.412 & $3.773^{*}$ & 0.068 & 2.567 \\
Shellfish & $34.037^{*}$ & $6.957^{*}$ & $6.691^{*}$ & $95.151^{*}$ \\
Finfish & 1.567 & 1.969 & $47.098^{*}$ & 0.759 \\
\hline
\end{tabular}

"Statistically significant at the 0.05 level.

week, but spend $\$ 0.14$ more on bacon, $\$ 0.30$ more for pork chops, and $\$ 0.09$ more on lambmutton per week than do households headed by whites. Households headed by individuals from other races spend $\$ 0.52$ less on ground beef, $\$ 0.27$ less on ham, and \$0.19 less on whole chicken, but spend $\$ 0.33$ more on other pork, $\$ 0.44$ more on shellfish, and $\$ 1.47$ more on finfish than do households headed by whites on a weekly basis. This result may be due to the fact that fish is usually in the regular diets of individuals of other races (e.g., Asians, Pacific Islanders).

Education is statistically significant in the ground beef, steak, bacon, pork chops, ham, other pork, whole chicken, chicken parts, and shellfish equations as indicated by the likelihood ratio tests. Specifically, households headed by college graduates spend $\$ 0.31$ less on ground beef, $\$ 0.28$ less on steak, $\$ 0.11$ less on bacon, $\$ 0.12$ less on pork chops, and $\$ 0.20$ less on ham per week, but spend $\$ 0.43$ more on other pork per week than do households headed by individuals with at most a high school degree. On the other hand, households headed by individuals with less than a high school diploma spend $\$ 0.15$ more on pork chops, $\$ 0.15$ more on other poultry, and $\$ 1.31$ more on shellfish per week but spend $\$ 0.31$ less on whole chicken and $\$ 0.25$ less on chicken parts per week than do households headed by individuals with, at most, a high school degree.
A nonlinear relationship exists between income and a number of meat products. In particular, expenditures on ground beef, steak, and pork chops increase with income initially before decreasing with further increases in income. On the other hand, expenditures on other pork, whole chicken, chicken parts, other poultry, shellfish, and finfish decrease with income initially before increasing with further increases in income. Income elasticities are presented in Table 5. These estimates are inelastic with the exception of shellfish.

The coefficient estimates of the variable $\Gamma_{\text {in }}$ are statistically significant in eight of the equations. However, the coefficient estimates of $\Gamma_{\text {ih }}$, except for other pork, are not statistically different from zero for the various beef and pork products. Consequently, deleting the observations corresponding to zero expenditure levels for the non-beef and non-pork disaggregate meat products would have introduced sample selection bias. However, deleting households with zero expenditure levels for the beef and pork products would not have biased the parameter estimates, although efficiency losses would have occurred.

\section{Concluding Remarks}

Most meat demand studies have focused on relatively aggregate commodities using either time-series or cross-sectional data. This article deviates from previous studies by focusing on 14 
Table 5. Income and Household Size Elasticities $^{\mathrm{a}}$

\begin{tabular}{lcc}
\hline Equation & Income & $\begin{array}{c}\text { Household } \\
\text { Size }\end{array}$ \\
\hline Ground Beef & 0.107 & 0.604 \\
Roast & 0.083 & 0.697 \\
Steak & 0.139 & 0.366 \\
Bacon & 0.044 & 0.810 \\
Pork Chops & 0.177 & 0.437 \\
Ham & 0.080 & 0.368 \\
Other Pork & -0.279 & 0.343 \\
Lunch Meat & 0.044 & 0.399 \\
Lamb-Mutton & -0.026 & 1.199 \\
Whole Chicken & -0.167 & -1.243 \\
Chicken Parts & -0.131 & -0.206 \\
Other Poultry & -0.206 & -0.184 \\
Shellfish & -1.260 & -1.243 \\
Finfish & -0.129 & 0.201 \\
\hline
\end{tabular}

${ }^{a}$ Evaluated at the means of variables.

disaggregate meat products using household microdata. In addition, due to the nature of the data, these 14 products are modeled in a systems framework based on the QES and estimated using a two-step censored regression technique.

The empirical results suggest that disaggregate meat expenditures are affected by various demographic factors. Moreover, the set of significant factors for each equation is not the same for each of the disaggregated products even among those with the same animal origin (e.g., ham, bacon, pork chops, other pork). For example, based on the likelihood ratio tests, regional and education factors significantly affect bacon, pork chops, and other pork expenditures. However, as a group, these factors are not statistically significant in the ham equation.

This study documents the importance of modeling meat expenditures at a disaggregate level. The expenditure patterns for disaggregate meat products enable various producer, processor, and consumer groups of the meat industry to identify key factors affecting household expenditures. Disaggregate analysis of meat products is useful for developing specific marketing programs because consumers do not necessarily purchase meat of the same type or animal origin (Nayga and Capps 1994).

Refinements and improvements in data collection are necessary to provide more definitive results for the meat industry. With advancement in computer technology and availability of supermarket scanner data, the analysis of meat demand at a disaggregate level is now more feasible than ever. A limitation of the present study is the lack of price data. The potential for demand analysis at the disaggregate level, using household microdata, will be enhanced if reliable and accurate price indexes are available for various disaggregate meat products. Research and market analysts should also lobby for more disaggregate information in government-sponsored surveys. Clearly, further analysis of disaggregate meat products merits attention.

[Received January 1995. Final version received February 1995.]

\section{References}

Belsley, D.A., E. Kuh, and R.E. Welsch. Regression Diagnostics, Identifying Influential Data and Sources of Collinearity. New York: Wiley, 1980.

Blaylock, J. and D. Smallwood. U.S. Demand for Food: Household Expenditures, Demographics and Projections. Washington, DC: United States Department of Agriculture, Economic Research Service, Technical Bulletin 1713, 1986.

Branson, R., H. Cross, J. Savell, G. Smith, and R. Edwards. "Marketing Implications from the National Consumer Beef Study." Western Journal of Agricultural Economics 11(1986):82-91.

Braschler, C. "The Changing Demand Structure for Pork and Beef in the 1970's: Implications for the 1980's." Southern Journal of Agricultural Economics 15(1983):102-10.

Buse, R. and L. Salathe. "Adult Equivalent Scales: An Alternative Approach." American Journal of Agricultural Economics 60(1978):460-68.

Capps, Jr., O. and R.M. Nayga, Jr. "Demand for Fresh Beef Products in Supermarkets: A Trial with Scanner Data." Agribusiness: An International Journal 7(1991):24151 .

"Effect of Length of Time on Measured Demand Elasticities: The Problem Revisited." Canadian Journal of Agricultural Economics 38(1990):499-512.

Chavas, J.P. "Structural Change in the Demand for Meat." American Journal of Agricultural Economics 65(1983):148-53.

Cheng, H. and O. Capps, Jr. "Demand Analysis of Fresh and Frozen Finfish and Shellfish in the United States." 
American Journal of Agricultural Economics 70(1988):53342.

Cox, T., R. Buse, and A. Alvarez. "Effects of Demographics on Changes in At-Home Meat Consumption." The Economics of Meat Demand, ed. R. Buse, pp. 217-24. Madison, WI: University of Wisconsin, 1989.

Cragg, J. "Some Statistical Models for Limited Dependent Variables with Applicaiions to the Demand for Durable Goods." Econometrica 39(1971):829-44.

Eales, J. and L. Unnevehr. "Demand for Beef and Chicken Products: Separability and Structural Change." American Journal of Agricultural Economics 70(1988):521-32.

Haines, P., D. Guilkey, and B. Popkin. "Modeling Food Consumption Decisions as a Two-Step Process." American Journal of Agricultural Economics 70(1988):543-52.

Heckman, J.J. "Sample Selection Bias as a Specification Error." Econometrica 47(1979):153-61.

Heien, D. and C. Durham. "A Test of the Habit Formation Hypothesis Using Household Data." Review of Economics and Statistics 73(1991):189-99.

Heien, D. and C. R. Wessells. "Demand Systems Estimation with Microdata: A Censored Regression Approach." Journal of Business and Economic Statistics 8(1990):365-71.

Lee, L. "Simultaneous Equation Models with Discrete and Censored Dependent Variables." Structural Analysis of Discrete Data with Econometric Applications, eds. P. Manski and D. McFadden, pp. 346-64. Cambridge, MA: MIT Press, 1978.
Menkhaus, D., G. Whipple, S. Torok, and R. Field. "Developing a Marketing Strategy for Branded, Low Fat, Fresh Beef." Agribusiness 4(1988):91-103.

Moschini, G. and K. Meilke. "Parameter Stability and the U.S. Demand for Beef." Western Journal of Agricultural Economics 9(1984):271-82.

Nayga, Jr., R.M. and O. Capps, Jr. "Tests of Weak Separability in Disaggregated Meat Products." American Journal of Agricultural Economics 76(1994):800-08.

"Away-from-Home and At-Home Beef Consumption in the United States: An Analysis Using Qualitative Choice Models.” Journal of International Food and Agribusiness Marketing 4(1992):61-81.

Nyankori, J. and G. Miller. "Some Evidence and Implications of Structural Change in Retail Demand for Meat." Southern Journal of Agricultural Economics 14(1982):65-70.

Pollak, R.A. and T.J. Wales. "Demographic Variables in Demand Analysis." Econometrica 49(1981):1533-51.

Pudney, S. "An Empirical Method of Approximating the Separable Structure of Consumer Preferences." Review of Economic Studies 48(1981):561-77.

Skaggs, R., D. Menkhaus, S. Torok, and R. Field. "Test Marketing a Branded, Low Fat, Fresh Beef." Agribusiness 3(1987):257-71.

Tobin, J. "Estimation of Relationships for Limited Dependent Variables." Econometrica 26(1958):24-36.

United States Department of Labor. Consumer Expenditures in 1992. Washington, DC: Bureau of Labor Statistics, Report No. 861, December 1993. 
http://www.jstor.org

\title{
LINKED CITATIONS
}

- Page 1 of 3 -



You have printed the following article:

Microdata Expenditure Analysis of Disaggregate Meat Products

Rodolfo M. Nayga, Jr.

Review of Agricultural Economics, Vol. 17, No. 3. (Sep., 1995), pp. 275-285.

Stable URL:

http://links.jstor.org/sici?sici=1058-7195\%28199509\%2917\%3A3\%3C275\%3AMEAODM\%3E2.0.CO\%3B2-7

This article references the following linked citations. If you are trying to access articles from an off-campus location, you may be required to first logon via your library web site to access JSTOR. Please visit your library's website or contact a librarian to learn about options for remote access to JSTOR.

\section{References}

\author{
Adult Equivalent Scales: An Alternative Approach \\ Rueben C. Buse; Larry E. Salathe \\ American Journal of Agricultural Economics, Vol. 60, No. 3. (Aug., 1978), pp. 460-468. \\ Stable URL: \\ http://links.jstor.org/sici?sici=0002-9092\%28197808\%2960\%3A3\%3C460\%3AAESAAA\%3E2.0.CO\%3B2-O \\ Structural Change in the Demand for Meat \\ Jean-Paul Chavas \\ American Journal of Agricultural Economics, Vol. 65, No. 1. (Feb., 1983), pp. 148-153. \\ Stable URL: \\ http://links.jstor.org/sici?sici=0002-9092\%28198302\%2965\%3A1\%3C148\%3ASCITDF\%3E2.0.CO\%3B2-E
}

\author{
Demand Analysis of Fresh and Frozen Finfish and Shellfish in the United States \\ Hsiang-tai Cheng; Oral Capps, Jr. \\ American Journal of Agricultural Economics, Vol. 70, No. 3. (Aug., 1988), pp. 533-542. \\ Stable URL: \\ http://links.jstor.org/sici?sici=0002-9092\%28198808\%2970\%3A3\%3C533\%3ADAOFAF\%3E2.0.CO\%3B2-8
}

\author{
Some Statistical Models for Limited Dependent Variables with Application to the Demand for \\ Durable Goods \\ John G. Cragg \\ Econometrica, Vol. 39, No. 5. (Sep., 1971), pp. 829-844. \\ Stable URL: \\ http://links.jstor.org/sici?sici=0012-9682\%28197109\%2939\%3A5\%3C829\%3ASSMFLD\%3E2.0.CO\%3B2-K
}


http://www.jstor.org

\section{LINKED CITATIONS}

- Page 2 of 3 -

\section{Demand for Beef and Chicken Products: Separability and Structural Change}

James S. Eales; Laurian J. Unnevehr

American Journal of Agricultural Economics, Vol. 70, No. 3. (Aug., 1988), pp. 521-532.

Stable URL:

http://links.jstor.org/sici?sici=0002-9092\%28198808\%2970\%3A3\%3C521\%3ADFBACP\%3E2.0.CO\%3B2-V

Modeling Food Consumption Decisions as a Two-Step Process

Pamela S. Haines; David K. Guilkey; Barry M. Popkin

American Journal of Agricultural Economics, Vol. 70, No. 3. (Aug., 1988), pp. 543-552.

Stable URL:

http://links.jstor.org/sici?sici=0002-9092\%28198808\%2970\%3A3\%3C543\%3AMFCDAA\%3E2.0.CO\%3B2-E

\section{Sample Selection Bias as a Specification Error}

James J. Heckman

Econometrica, Vol. 47, No. 1. (Jan., 1979), pp. 153-161.

Stable URL:

http://links.jstor.org/sici?sici=0012-9682\%28197901\%2947\%3A1\%3C153\%3ASSBAAS\%3E2.0.CO\%3B2-J

\section{A Test of the Habit Formation Hypothesis Using Household Data}

Dale Heien; Cathy Durham

The Review of Economics and Statistics, Vol. 73, No. 2. (May, 1991), pp. 189-199.

Stable URL:

http://links.jstor.org/sici?sici=0034-6535\%28199105\%2973\%3A2\%3C189\%3AATOTHF\%3E2.0.CO\%3B2-\%23

\section{Demand Systems Estimation with Microdata: A Censored Regression Approach}

Dale Heien; Cathy Roheim Wessells

Journal of Business \& Economic Statistics, Vol. 8, No. 3. (Jul., 1990), pp. 365-371.

Stable URL:

http://links.jstor.org/sici?sici=0735-0015\%28199007\%298\%3A3\%3C365\%3ADSEWMA\%3E2.0.CO\%3B2-M

\section{Tests of Weak Separability in Disaggregated Meat Products}

Rodolfo M. Nayga, Jr.; Oral Capps, Jr.

American Journal of Agricultural Economics, Vol. 76, No. 4. (Nov., 1994), pp. 800-808.

Stable URL:

http://links.jstor.org/sici?sici=0002-9092\%28199411\%2976\%3A4\%3C800\%3ATOWSID\%3E2.0.CO\%3B2-8 
http://www.jstor.org

\section{LINKED CITATIONS \\ - Page 3 of 3 -}

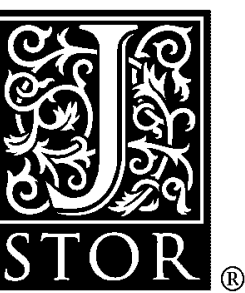

\section{Demographic Variables in Demand Analysis}

Robert A. Pollak; Terence J. Wales

Econometrica, Vol. 49, No. 6. (Nov., 1981), pp. 1533-1551.

Stable URL:

http://links.jstor.org/sici?sici=0012-9682\%28198111\%2949\%3A6\%3C1533\%3ADVIDA\%3E2.0.CO\%3B2-T

An Empirical Method of Approximating the Separable Structure of Consumer Preferences S. E. Pudney

The Review of Economic Studies, Vol. 48, No. 4. (Oct., 1981), pp. 561-577.

Stable URL:

http://links.jstor.org/sici?sici=0034-6527\%28198110\%2948\%3A4\%3C561\%3AAEMOAT\%3E2.0.CO\%3B2-H

\section{Estimation of Relationships for Limited Dependent Variables}

James Tobin

Econometrica, Vol. 26, No. 1. (Jan., 1958), pp. 24-36.

Stable URL:

http://links.jstor.org/sici?sici=0012-9682\%28195801\%2926\%3A1\%3C24\%3AEORFLD\%3E2.0.CO\%3B2-R 